PROLEGOMENA TO PROBLEMOLOGY: A Definition of Social Problems

\author{
By Paris Arnopoulos \\ CONCORDIA UNIVERSITY \\ Montreal \\ 1995
}

\title{
TABLE OF CONTENTS
}

INTRODUCTION: A World of Problems.

\section{CONCEPT}

1. FORM: Anatomy of Problems.

2. FUNCTION: Meaning of Problems.

\section{STRUCTURE}

1. CONTEXT: Topology of Problems.

2. CONTENT: Topicality of Problems.

\section{PROCESS}

1. PERIOD: Problem Timing.

2. METHOD: Problems-Solving.

CONCLUSION: The Global Problematique.

Background Bibliography 
It is by now rather banal to affirm that the 21 st century world will be undergoing a critical period of transition in which a multitude of problems is accumulating. All around us, one can see that things are not evolving as they should. Of course, such perceptions and sentiments have been expressed many times throughout history. The world has always been changing and this change has not often been satisfactory to many people. Crises and problems, therefore, are nothing new in the lives of men and nations.

Yet, there is something new both in the quantity and quality of contemporary problems. For the first time in history, the world is a single mega-system; with a multitude of interacting and interdependent units. We, therefore, live in a global system, as never before. Because of that, the mass and kind of problems we face are also interacting and interdependent. The increased magnitude and complexity of our creations have produced more and bigger problems than ever. So, not only has the number of our problems grown beyond anything known before, but their novelty and complexity are taxing our ability to understand and manipulate them. It seems that current events are becoming too large and are moving too fast for us to grasp and control. Human actors and institutions appear increasingly diminutive in relation to the flow of events.

Undoubtedly, mankind has always been at the mercy of nature. The laws and forces of the universe inexorably dominate human existence. But again, this is the first time that man's own works have reached such magnitude as to overshadow the problems posed by nature. Science and technology have advanced to the point where they seem to have taken a life of their own and are moving by their proper momentum. What is worse, their effects upon their creators, both individually and collectively, were not foreseen; hence, their repercussions upon our lives are both dramatic and traumatic.

The uniqueness of our era consists of the growing dominance of artificial over natural problems. Human accumulation of power means that we are now capable of creating and destroying many things, including ourselves. Unfortunately, our destructive capacity is out-pacing our creative one; so the threats to humanity become more awesome than the promises. So, problems multiply faster than we are able to solve them and the lead-time for making decisions shrinks into nothingness.

On the basis of this rather pessimistic overview, it behoves us to take things seriously and see what we can do to understand the problems of this world so that we can improve our capacity to solve them. Even if problems are intrinsic to any open and dynamic system; controlling them can make the difference between life and death. Anyone who can contain problems within a functional range can turn them to advantage in developing better systems. Thus, before we can think of sustainable development, we must conceptualize the global problematique.

With that as an incentive, this study initiates a systematic definition of problems as the first step in an emerging problemology. In the following presentation, we can only scratch the surface of this large area and leave for another time an in-depth research into the subject. This beginning is a prerequisite to any scientific treatment of problems, because it lays down the foundations for further work. Here then, we outline the concept, structure, and process of problems and by doing so answer the questions related to the nature, meaning, type, location, time, and solution of our critical issues.

Accordingly, the paper is divided into three parts of two chapters each, covering successively the systemic, static and dynamic aspects of problems. The chapters deal with the form and function, content and context, as well as period and method of this subject. Thus, although the study does not go into any depth; it does cover the field of problem analysis exhaustively and sets the basis for more detailed and specialized research later on. 


\section{CONCEPT}

At the beginning of any systematic study of a subject, one must formulate the basic concepts which define the nature and scope of the topic in question. This conceptualization of the subjectmatter explicates what exactly is to be studied and why. The answer to these two questions should provide the essence and meaning of the study; thus, placing the research which follows in its proper perspective.

In this case, the topic under investigation is "problems," so we first have to define the term in quotation marks and then explain what it means. This undertaking will give us the form and function of the term and therefore develop its conceptualization. The following two sections treat each of these aspects in turn.

FORM: Problem-anatomy.

The first question which must be answered before going any further is: what is a problem? Obviously, to begin with, this is a matter of definition, and as such it is both easy and difficult to do. It is easy because definitions are arbitrary and a priori conceptual devices, so anything can be defined in any way. Yet, to facilitate communication, one must respect popular and traditional meanings of common sense, so we fit-in our definition to generally acceptable standards.

Because "problem" is such a term, it means many different things depending on who is using it and under what circumstances. For this reason, there are several definitions of the term at various degrees of conceptual formality. We have chosen to formulate the following definition because it combines simplicity and suitability for our purposes: i.e. a problem is a disturbing situation.

Although this definition could apply to any problem, it is best suited to our context because it emphasizes the situational nature of the problems with which we are particularly concerned. By situation we mean a specific condition or state of affairs which draws attention to itself. Situations, therefore, are salient points upon which interest is focused and as such describe the essence of problems. In this sense, real problems of this world are disturbances in the various configurations of matter-energy within space-time.

Since we are not interested here in symbolic or semantic problems, the above meaning will do very well to concentrate our study to empirical problems of human concern. We are, therefore, excluding from our purview mathematical questions, intellectual puzzles, or psychological malaises, although they are problems of sorts, instead focusing our attention to actual external events which disturb us. It is in this sense that we propose to study worldly problems of current affairs.

Undoubtedly, there are many such problems in the contemporary world. So much so that to many people they seem to be infinite or indefinite. Yet, the Encyclopaedia of World Problems \& Human Potential has hunted down and classified almost 10,000 world problems, identified and documented by 15,000 INGOs. In a different project, the US Library of Congress identified 1,200 world problems, but another independent study only produced 50 global problems. Finally, the Rio Conference discerned 40 fundamental problems which must be solved by the year 2000 as part of its Agenda 21.

Playing with numbers, of course, one can come up with any figure; since it all depends what categories and levels of abstraction or aggregation one uses for the list of problems. Whether they are counted in tens or in thousands, what is important is that the disturbing situations in the world are recognized and dealt with. But for this to happen, we must find out as much as possible about them from all points of view. It is this process of problem-analysis that we are performing here in broad terms.

Basing our problem-analysis on the above definition and elucidation given so far, we now proceed to outline six fundamental parameters common to the problems we are dealing with: 
a) Situational reality: the form of the problem in question and its concreteness or abstractness in operational terms.

b) Systemic functionality: the objective possessor of problems as the normative measure of disturbances.

c) Contextual relativity: the scope and perspective of the problem which qualifies its extent and relationship with other situations.

d) Reflective subjectivity: the awareness of a problem by somebody and the impact it has on sentient beings.

e) Dynamic chronology: the change it undergoes through time and the causality of antecedents and consequents.

f) Soluble potentiality: the optimal method according to which an intelligent system can deal with the problems presented to it.

A properly defined problem must contain information on all of the above six dimensions. On that basis one can distinguish between true and pseudo-problems. As an example, for "poverty" to be determined as a problem, it must be: first, operationally defined; second, functionally explained; third, contextually placed; fourth, relevantly specified; fifth, historically developed; and sixth, probably solvable. Only then should it be considered as a rigorous problem to be dealt with in a systematic manner.

Very few empirical problems, of course, can be so completely specified in all their aspects to be scientifically impeccable. For this reason, most problems are debatable. In that case, when the parameters of problems are moot, we speak of "issues". A public issue, then, is joined to clarify the above dimensions and thus determine the quality and quantity of given problems. As in this opening section we have presented the situational aspects of problems, each of the following sections will deal with the remaining five aspects in greater length.

\section{FUNCTION: Problem-meaning.}

According to our definition, problems are "disturbing situations." So far, we have explained the "situational" aspect of the definition; now, we do so for the "disturbing" aspect. To begin with, a disturbance is an extraordinary or abnormal stimulus which could upset the steady-state of a system. It is thus an incident creating some dissonance, contradiction, discrepancy or imbalance in a situation. So qualified, a problematic condition becomes an abnormal state which presents a potential threat to its subject.

This description of a problem requires some explanation and the best way to do that is by systems-analysis. As we used the term "system" above, we meant a set of interrelated-interacting units. This implies the existence of an ordered group of elements undergoing a dynamic process in space and time. This systemic process is evident throughout the universe and manifests itself similarly throughout atomic, molecular, biological, human, or social activities. In whatever level one cares to look, "reality" is a conglomeration of such dynamic and open systems.

The essence of a system is some underlying order in its being. It is this order which distinguishes a system from chaos: i.e. a random agglomeration of things in erratic movement. Accordingly, we can postulate that problems cannot exist in chaos; They can only be found in systems. This is because a problem relates to a change in the order of things; without some such order, it would be nonsense to speak of problems.

The next step of this reasoning is that an order implies a norm: i.e. some standard or principle according to which a system usually operates. In this sense, there is a normal condition of the system which a problem could disturb by introducing an abnormal element into it. The threat is that in extreme cases the disturbance may escalate to the point of breaking down the system altogether. For the sake of self-preservation, therefore, a system must be able to contain its problems and maintain its normal steady-state operation. 
For example, 'starvation' is a problem for a healthy system because, in the first instance, it lowers the organism's normal performance and ultimately may hasten its death. However, starvation would not be a problem to an obese or abnormal organism; on the contrary, it may prove to be the solution.

From these examples, we can see that a problem is whatever interferes with the proper functioning of a system by impeding its optimal performance. Clearly, "proper" and "optimal" are normative criteria of systemic function, against which a problem can be measured: the more this function is disturbed, the greater the problem.

In this sense, a problem may be described as a malfunction of a system from its normal or ideal state. If a system is not working as it should be, there is a problem. Thus, another way of perceiving a problem is to look upon it as a gap between facts and values; a difference between what is and what ought to be: the wider the gap, the bigger the problem.

This way of measuring the magnitude of a problem implies that we know both the real and ideal conditions of a system, something which is not always so easy. Very often we may be sure that there is something wrong with a particular situation but cannot say exactly why, because we are unable to formulate the norm upon which we judge the discrepancy. The condition of general malaise is an example of such incapacity to specify our desiderata.

By knowing at least the approximate position of normality, one can diagnose the situations beyond it. What lies outside the normal may be described either qualitatively or quantitatively. A quantitative problem is simply a situation of insufficiency (i.e. deprivation or underdevelopment) or excess (i.e. obesity or overdevelopment); whereas a qualitative problem is a more complex condition of the wrong kind of make-up (i.e. malnutrition or maldevelopment). Having too much, too little, or an improper combination causes problems in any system (organic or social). A problem, therefore, may be an imbalance between what a system has and what it needs to function properly. That is why, problems of human needs arise when there is hunger or disease.

If norms are well known, they become ideals: i.e. the goals which the system tries to achieve or maintain. In that case, when the goal of a system is set; the only problems that may arise are those of means or strategy. These are scientific and technical problems, where gaps between knowledge and ignorance are relatively well defined. Cancer or metal fatigue are such problems in which doctors and engineers are working on.

In contrast, poverty or mental fatigue are sociological or psychological problems where norms or ends are either unknown or conflicting. Poverty is a uniquely human social insufficiency syndrome which has no meaning beyond mankind. So, when values and goals are non-existent or contradictory, problems become much more complex and difficult. For that reason, problems of exploitation or disorientation are so insidious. Most of these problems may be endemic to the human condition, so they persist through history.

Because they are disturbing situations, problems tend to acquire some negative connotations. Since they are a threat to the wellbeing of a system, problems are to be avoided. It is as if nature abhors problems! And yet, problems can be quite functional in the development of systems. As gaps or imbalances, they present a challenge and so an opportunity for action and correction. Without problems, a system would have little incentive to progress and adapt to changing conditions. Problems can thus serve as stimuli to improve the performance of systems and develop new and better strands.

In that sense, problems are both necessary and desirable in an imperfect and evolving world. By acting as sinks or black holes, they absorb any excess energy which may be generated by dynamic processes. For this reason, it has been suggested that problems be institutionalized or builtinto systems as part of their cybernetic mechanism. Be that as it may, the unavoidability of problems forces us to try and understand them so that we may use them for our purposes. We, therefore, continue our search on the different aspects of problems on the next chapter. 


\section{STRUCTURE}

Based on the problem-conceptualization just completed, we now go on to outline the fundamental structure of problems. Structuring a problem means locating its spatial context and identifying its human content. Problems do not exist in vacuum. They are to be found within a certain place and relate to particular things. Therefore, the analysis of a problem must be able to answer the questions of where and who. This is what we intend to do in the two sections of this chapter.

CONTEXT: Problem topology.

Real problems take place somewhere in this world. A situation occurs some place far or near, large or small. A problem can, therefore, be located by space and size: two characteristics that would constitute its scope. These two measures may be ranged within a continuum whose extremes are micro and macro problems. Towards the micro end would be found individual and local problems, whereas towards the macro end would be global and universal ones.

According to this scheme, we classify problems within three main areas: local; regional; and global. Local problems take place in relatively restricted areas or spaces, all the way from an estate to a society. Within this range are found problems involving families, groups, communities, nations, or a combination of these. At the next level of magnitude, are located regional problems involving groups of nations, continents or blocs. The problems of the first, second and third world nations respectively would be placed within these areas. Finally, at the highest level of generality are global problems involving mankind as a whole. These problems could be transnational, interregional or planetary, including extra-terrestrial.

The tabulation in the next page contains the type of problems which could be placed in the various categories we have created, based on the criterion of increasing geographical extent. Reading from top to bottom, the table begins with the micro-problems of local communities and ends with the macro-problems of the world as a whole. Examples of what we consider significant problems in each area have been given at each level.

The representative problem chosen for each level shows the nature of worldly problems as imbalances of some sort. The problem of interference, for example, be it one of the individual versus the state or local autonomy versus world government, involves the question of how entities coexist and cooperate without getting in each other's way. The breakdown of local communities, the rise of the national, state and international conflicts, are all different aspects of the frictions and contradictions among various geographical units.

At the continental level, are the problems concerning an entire culture at a certain point of its development. These problems differ for each cultural area; the major problems of one are not likely to be those of another. Thus, the problems of preindustrial, industrial and post-industrial societies are not the same. The western world has problems of overdevelopment, whereas the third world suffers problems of underdevelopment; with the second world having those problems peculiar to industrial economies.

Finally, at the global level, problems cross-cut national and cultural boundaries to extend throughout the world. Problems involving transnational activities, east-west cultural confrontation and north-south income gap, are such examples. At the extreme, the issues of outer space and the high seas, as well as the general problems of world government, environmental pollution and resource depletion encompass the whole earth as a unit.

The increasing interdependence of the world makes all these problems more and more interrelated, both horizontally and vertically: local problems may eventually become global and western ones may become eastern. In spite of this overlapping, this geographical delimitation of problems is useful for analytic purposes. 
CONTENT: Problem-subjects.

As already stated, real problems can only exist in relation to an existing system. A problem must belong to somebody or something. This means that in addition to its location, a problem requires a subject or a theme of activity to which it has a functional relationship. In this sense, we speak of a machine, an organism, a society, or the world having problems because there is a malfunction somewhere in their operation. For this to happen, we assume that these systems have objectives or purposes which the problem hinders from achieving.

Although systems may have intrinsic purposes, in which case they may have objective problems; very often it is human beings who assign them goals, which if they cannot easily attain are considered problematic. In this sense, problems are in the eyes of the beholder as much as in the object itself. This juxtaposition of objective and subjective conditions means that a problem is not only a "state of affairs" but also a "state of mind."

Without taking a solipsistic position, one can admit that there is some truth to the subjectivity of problems, especially in human affairs. Since we are particularly concerned with the problems of this world, one may easily make "man the measure of all things" and look at problems from his point of view. On the assumption that humanity is our system, everything else becomes the environment. Thus, problems can either be internal to the system or external between the system and its environment. In the former case, they are personal problems whereas in the latter, they are either social or natural.

The tabulation in the following page elaborates on this distinction. Evidently, that table uses the same conceptual framework as the previous one in its classification of problems. We have retained this uniform trinary taxonomic scheme to facilitate comparison and symmetry.

Accordingly, our contextual classification begins by dividing human reality into three broad areas: personal, social and natural. By extension, problems fall into one or another of these categories, depending on whether they involve the internal or external worlds of mankind.

Personal problems are inner disturbances or imbalances of human beings. These produce a feeling of discontent or dissatisfaction accompanied by a state of tension and instability. They are incongruities between man and himself, between ego and id, mind and soul. More specifically, personal problems may be grouped under spiritual, intellectual or psychological headings, with some examples given for each one. It should be noted that all cases assume a certain functional norm the denial of which creates a problem (for that reason, "superstition" rather than "truth" is identified as a problem in general terms).

The next large area of problems falls under the "social" label. This type of problems are disturbing situations among people, rather than within them. They are the problems of "man's inhumanity to man", the disorder in interpersonal relations. These problems are manifested in the cultural, political and economic spheres of action, as in the cases of social anomie, power insecurity or wealth inequality. As the examples in the table show, social problems are in the heart of world affairs and relate to both personal and natural dysfunctions.

The latter reflect the imbalances between culture and nature. Humanity, of course, is part of nature and as such cannot escape its laws with impunity; if we do not live according to certain standards, we have problems of health: physiological or psychological. Naturally, no matter what we do, we eventually die. Death is part of the general law of entropy which all things seem to follow in the long run, so it cannot be considered as an objective problem even if life and order try to prolong themselves and postpone their inevitable degradation.

Having given the locational and contextual aspects of human problems, we can conclude this chapter by combining these two dimensions into a single framework. The matrix in the following page has been constructed by using the spatial classification in the vertical and the thematic in the horizontal position. In this manner, the two schemes intersect each other and the nine categories of each one produce eighty-one composite groups of problems.

This two-dimensional classification gives a more precise location of problems than either of 
the previous one-dimensional lists. Some of the examples given before have now been reproduced in this table to show their position in relation to the combined criteria. Thus, "underdevelopment" is an economic problem to be found mainly in the third world; whereas the uneven distribution of natural resources creates problems of interregional or interdependent relations.

Apart from the relative arbitrariness of any classification scheme; it is to be noted that the reason for the absence of examples in some of the boxes may be either objective (there are no problems therein) or subjective (none can be found there).

Be that as it may, our problem structuring shows their relativity: what may be problems in one area for some people are quite normal conditions in another place for others. So, an individual problem is not necessarily a systemic one, and vice versa. Therefore, the analysis of problems must specify as much as possible whose and where the problem belongs.

Oppression, for example, affects the oppressed differently from the oppressor, although it may be a problem for both. Similarly, undernourishment is found only in certain areas and affects certain people; whereas in other places and for other people the opposite problem of overnourishment is more common. It is on the basis of this knowledge that one can determine the extent of the problem and thus define its structure both in terms of context and content.

\section{PROCESS}

This last chapter of problem-analysis deals with the process by which problems evolve or change. This stage of the study follows logically that of structure, because we now move from the static to the dynamic aspects of problems. Given the way problems were conceptualized and the compartmentalized, we presently try to find some temporal and causal relationships among them. Consequently, we are introducing the element of time and variation into our subject-matter in order to answer the when and how of problems. The following two sections, thereby, treat the chronological and methodological aspects of this topic.

PERIOD: Problem-Timing.

Our reality exists in space and time, so real problems are to be found within this framework. As problems take place in a certain location, so they happen at a certain period. The arrow of time may be conceived as a vector of change relative to the constant movement of matter and energy. In existential, open and dynamic systems, problems arise when particular movements tend to deteriorate the structure and functions of the system.

From this dynamic point of view, problems are directly associated with change. More precisely, we might say that a problem occurs whenever a situation calls for change. Problems arise whenever something must be done; otherwise there are no problems. A status quo which suits the system, presents no problems and does not require any changes.

A situation of optimal homeostasis is not disturbing and presents no problem. Acceptable changes or events are not problems; it is only when the flow of things or the current situation becomes unacceptable that a problem develops, because it is then when something must be done about it.

Since problems "take time" and involve change, we can classify them on two criteria: timing and duration. The former tells us when a problem happens and the latter how long it lasts.

Obviously, problems vary in both these dimensions; so we can construct two continua to measure their occurrence. The first one divides problems in three major periods:

a) Past: $\quad$ Problems of historical interest (ancient or modern);

b) Present: Problems of current events (contemporary or pending);

c) Future: Problems of potential outcome (prospective or probable).

Problems change in time. Yesterday's problems are not the same as today's and most likely will not be the same as tomorrow's. Many historical problems which were very crucial once have 
now become obsolete and forgotten; while new ones have taken their place.

Nevertheless, not all problems come and go; some remain with us for a long time, even forever. The life of problems, therefore, varies from quite short to very long. On this criterion, we divide problems into three main types:

a) Ephemeral: Temporary problems (momentary or incidental);

b) Periodic: Recurring problems (cyclic or repetitive);

c) Eternal: Perennial problems (endemic or inherent).

Real problems, of course, may straddle these categories in various ways. Some problems which were thought of as obsolete may rise again to haunt us. Others which are considered eternal may disappear with changing circumstances. It is not easy to say which problems are part of the human condition and which are only part of human fashion.

In any case, we are not going into further details or more elaborate classification of problems and their examples, (the reader can provide some from one's own experience). What we will do is show how the temporal aspect of problems can be combined with the spatial and contextual dimensions to produce a new relationship among them. The matrix presented in the following page illustrates this space-time relationship.

The fundamental structure of this matrix is the same as that of the previous chapter. The horizontal dimension representing subject and vertical space levels. The visible difference here is that this is a $3 \times 3$ matrix, whereas the other was a 9x9 one. This means that we have simplified our categories down to those mentioned and omitted the other ones, in order to focus on those areas of particular interest to world affairs.

On that basic framework, we superimposed two other criteria. The horizontal is that of urgency. This indicates the priority of a problem. Urgency is directly proportional to immediacy: the more urgent, the more prior a problem is. It seems that in our world, the most urgent problems are economic ones and the least urgent political. According to diplomatic activity, the north-south economic problem has replaced the east-west political problem as the most immediate issue of world affairs.

The vertical dimension measures the importance of problems; which is to say the depth to which they affect people. The most important problems are those closer at hand, whereas the least important are those far away. According to survey polls, local or national problems come first; whereas, international or global ones' trail last. Combining all these measures, we have the typology shown in the matrix. The nine examples correspond to the different possible permutations among these variables.

On the other hand, it may be said that from the point of view of the global system and the long-run, the significance or importance of problems is the reverse of that shown in the matrix. From the broad and ultimate perspective, it is our treatment of the global-political problems that will determine the existence and shape of our world.

This is particularly so as historical evolution is accelerating. To the extent that change becomes more rapid and more radical (i.e. revolutionary), our reactions become more urgent (i.e. critical). Thus, as our lead-time is shortened, we have to look further and wider in time and space in order to survive the rising and spreading of problems.

\section{METHOD: Problem-Solving.}

Closely associated to problem chronology is the process through which they evolve. From this point of view, a problem goes through various stages of development, the most important of which are:

a) Formative: The causal ingredients or factors creating a problem;

b) Critical: The juncture at which the problem is at its most acute;

c) Anticlimactic: The post-phase of chronic or solved problems.

The formative stage of a problem, of course, is very significant because it contains the seeds of the disturbance. If we want to prevent problems, it is at this stage that we must prepare to act. 
This presupposes that one become aware of the developing problem before it reaches the critical stage. Very often the factors which cause a problem are beyond our control; therefore, the only thing one can do is prepare to face the problem when it comes. It may be however, that by the proper intervention, the course of events changes and the problem is prevented. In that case, a bit of timely action at an early stage may save much greater effort later. Knowing the difference between avoidable and unavoidable problems is thus crucial.

If a problem is inevitable, then it reaches a stage when the need for action is at its highest. Since, per definition, problems are disturbing situations, they act as stimuli which draw attention to themselves. This stimulus breaks our complacency and forces us to do something to re-establish an equilibrium. Problems give us the feeling that there is something wrong somewhere. This is an unsatisfactory and hence unacceptable condition which one tries to correct. This seems to be the way in which the need to solve will satisfy us and fulfil the demand for harmony within or outside ourselves. Problems are things which bother us; therefore, they prod us to act. If something can be left alone, it is not much of a problem.

From what has been said, it would appear that problems call for solutions. Although, not all problems are necessarily solvable, one at least tries to solve them by searching for a way out. It is indeed unusual if one is disturbed by something and does not attempt to change that situation. Thus, we may say that a solution is the change required to correct a disturbing condition. Accordingly, problem-solving is a process which attempts to transform an unacceptable situation into a satisfactory one.

As a result, a systematic sequence of such problem-solving process would go as follows:

a) Science: The prerequisite information and knowledge about the problem and its parameters (problem-analysis);

b) Policy: The sufficient intention to do something about the problem and find a way out of it (solution-strategy);

c) Action: The necessary operation to implement the problem- solving policy and satisfy the need (treatment-execution).

These three areas of activity are indispensable in any rational problem-solving exercise. To begin with, a problem must be perceived by someone, it must be defined and then studied as to its causes and effects. Only by knowing as much as possible about a problem can one hope to solve it correctly. Once this is done, one can evaluate the gravity of the problem and then prescribe a solution. This may be a plan of action or program of cure to be followed. With such intended plan at hand, all that is left is the actual carrying it out. For complex problems, of course, this means organizing and availing the necessary resources to do the job, as well as undertaking the operation to overcome the obstacles and reach the goals set for the solution.

Problem-solving, therefore, requires a capacity to perform various tasks: semiosis, aetiology, analysis, diagnosis, prognosis, therapy, logistics, cybernetics and praxis. Evidently, one does not go through an algorithm for everyday problems which are often handled instinctively or traditionally. But, as systems become more complicated, so does the problem-solving process. Simplistic solutions to complex problems not only are ineffective but tend to aggravate an already disturbed situation. So, certain technological efforts to buffer human activities from small disturbances may make them more vulnerable to large-scale catastrophes.

In this connection, we must say that problems themselves form systems. Complex systems tend to produce a chain of problems which are interrelated in the same way as the system to which they belong.

The UIA study already mentioned clustered its 10,000 world problems into 320 overlapping hierarchies, linked by 120,000 relationships of seven types.

Moreover, their preliminary search along nine million pathways identified seven thousand vicious circles of seven problems each. A cycle is a chain of problems, each of which aggravates the next, with self-sustaining positive feedbacks which carry the loop to its ultimate destruction. Simple loops start with three problems, but complex ones go much higher. 
This episystem or "problematique", has the following traits:
a) Multiplicity:
Large number or mass of problems;
b) Gravity:
Deep seriousness or weight of problems;
c) Complexity:
Great intricacy or difficulty of problems;
d) Universality:
Wide spread or omnipresence of problems;
e) Rapidity:
Fast accelerating evolution of problems;
f) Interactivity:
Manifold interdependence among problems.

Given this problematic situation of complex systems, problem-solving becomes a difficult enterprise which requires increasing time and effort to be done properly. It is for this reason that the science of problems, or problemology, should be developed at this time.

So far, in the relatively simple world of the past, problems could either be left alone to solve themselves or tackled haphazardly and in isolation. Presently, however, such laissez-faire attitude is no longer adequate to maintain our systems in working order. Unresolved problems create positive feedbacks which worsen the situation and eventually get out of control.

Hence, a systemic problem-solving methodology must be based in an integrated systemstheory to avoid solving one problem here and now by creating more problems elsewhere later on. The cross-cutting, cause-effect repercussions among problems should make us very careful as to what constitutes a real solution and not simply another problem.

The survey mentioned in the first chapter, identified 6,000 problems which aggravated each other, while only 300 which alleviated each other. With such dangerous relationships, problemsolving becomes a delicate and elusive undertaking. Yet, it is for precisely these reasons that it must be seriously engaged and methodically executed.

\section{CONCLUSION: The Global Problematic}

Since the presentation of this study has been very succinct, we need not summarize it any further at this point. The six parameters of problem-analysis, grouped into three dyads (concept; structure; process) should be clear enough not to require prolonged elaboration. Of course, each aspect could be studied in depth and practically applied to actual problem-cases by exhaustive research. Hopefully, this will be done by others; but it is not the purpose of this report.

What we will do here in the conclusion is to take a synoptic view of the problem-system in the world scale and see how our categories apply to it. As already mentioned, this problem-system has been labelled the "global problematique" and denotes the contemporary situation confronting humanity. This condition is characterized by an intricate and dynamic complex of dysfunctions making up the problem-system.

Following our typology, we can now classify these dysfunctions into six areas:

a) Formal: The web of disturbances produce a mega-problem of global

dimensions, thus increasing the system vulnerability.

b) Functional: The lack or clash of values and goals for individuals and collectives increases the disorientation of the system.

c) Topical: The complexity of many system variables increases risks of feedback and difficulties of solutions.

d) Spatial: The great asymmetries of wealth and power of world actors increases dissatisfaction, friction and conflict.

e) Temporal: The rapidity of historical change and technological innovations bring about a storm of crises and revolutions.

f) Procedural: The magnitude and novelty of the problems outpaces and outmodes methods and institutions of problem-solving. 
In other words, the world-system has become more vulnerable, unstable, asymetric and revolutionary, at the same time as its concepts, structures and processes for handling these problems have become outdated or obsolete. The different rates of change have created a widening gap between values, regions, disciplines and institutions, thus increasing the instability of the system. This means that the discrepancies between problems and solutions have been increasing.

The inadequacy of our capabilities or perhaps unwillingness to handle world problems may be illustrated in the following statistics taken from the study already mentioned. The over 1,000 multilateral treaties in force currently, only treat about 250 world problems. The 700 human values accepted today only relate to 350 problems. The 300 Inter-Governmental Organizations and 3,000 Non-Governmental Organizations operating throughout the world are only handling 300 and 700 problems respectively. Finally, about 2,000 disciplines in the world only study about 1,300 problems. When we recall that the grand total of the problems identified was approximately 10,000, these figures show how insufficient are our means for treating the illnesses of our system.

Nevertheless, there is considerable human potential which could be brought to bear in solving world problems. So far there have been identified 1,400 spiritual and mental potentials and 3,000 awareness modes which could help human development to meet these problems. Together with 230 clusters of dual polarities (good-bad), or 1,000 positive-constructive and 2,000 negativedestructive human values, the world disposes of several thousands of strategies used by international bodies to respond to world problems and promote a better future.

No matter how misleading statistics may be, there is no question that the growth in quantity and quality of world problems is happening at a faster rate than the growth of our mechanisms for their solution. Before this gap becomes unbridgeable, if it has not already reached that stage, there is a pressing need for improving our abilities for problem-analysis and problem-solving. Once we have this increased knowledge, we may hopefully acquire the necessary resolution to apply it in the service of the world. Hopefully, this short study has contributed in this long task. 


\section{BACKGROUND BIBLIOGRAPHY}

R.L. Ackoff: Redesigning the Future, John Wiley, N.Y. 1974.

J.L. Adams, Conceptual Blockbusting, Stanford, 1974.

P.J. Arnopoulos, Conceptual Framework of Human Concerns.

Report to BEP, Unesco, Paris, 1979.

Sociophysics, Nova Science, N.Y. 1993

Sociopolitics, Guernica, Toronto, 1995

B.E.P. Problem Analysis \& Table of Objectives for Medium-Term Plan. Unesco, Paris, 1974.

A. Birenbaum \& E. Sagarin (eds.) Social Problems, Scribner, N.Y. 1972.

D. Braybrooke, Philosophical Problems \& Solutions, Collier-Macmillan, Toronto, 1965

R. Brody: Problem-Solving. Human Sciences Press, N. Y. 1982

S. Carnarius, Management Problems \& Solutions, Addison-Wesley, Reading, Mass., 1976.

E.P.R.C. Contemporary Societal Problems, S.R.I. Menlo Park, 1971.

C. Eden: Messing About in Problems. Pergamon, N. Y. 1983

R. Eyestone: From Social Issues to Public Policy, Wiley, N.Y. 1978.

F.E. Emery (ed.) Systems Thinking, Penguin, Middlesex, 1969.

A. Gabus \& E. Fontela, Perceptions of the World Problematique. (DEMATEL Report). Battelle Institute, Geneva, 1975.

S. Gordon, World Problems. Batsford, London, 1971.

R.L. Henshel, Reacting to Social Problems, Longman, Toronto, 1976.

R. Havelock \& A. Huberman, Solving Educational Problems.

UNESCO, Paris, 1977.

J.L. Henderson, World Questions, Methuen, London, 1963.

A. Judge, Encyclopaedia of World Problems \& Human Potential.

Union of International Associations, Brussels, 1994.

C. E. Lindblom: Usable Knowledge. Yale U. P. N. H. 1979

K. Mannheim: Diagnosis of our Time. Routledge, London, 1943

R.K. Merton, Contemporary Social Problems. Harcourt-Brace, N.Y. 1971

D. N. P. Murthy: Mathematical Modelling. Pergamon, N. Y. 1990

J. Rachels. Moral Problems. Harper \& Row. N. Y. 1971

P. N. Rastogi: Policy Analysis \& Problem Solving, Sage, N. P. 1992

E.F. Shumacher: The Guide for the Perplexed,

Fitzhenry \& Whiteside, Toronto, 1977.

Secretariat, List of Social Concerns. O.E.C.D. Paris, 1973.

F. Vidal, Problem-Solving Methodology. Dunod, Paris, 1971.

P. Worsley, Problems of Modern Society. Penguin, London,1972. 